\title{
A LOW PREVALENCE OF RAPIDLY PROGRESSIVE INTERSTITIAL LUNG DISEASE IN LATIN AMERICAN PATIENTS WITH DERMATOMYOSITIS: A MULTICENTER CROSS-SECTIONAL STUDY
}

\author{
Pablo Arturo Olivo Pallo, ${ }^{1, *}$, Leonardo Santos Hoff², Jiram Torres², Amelia Granel ${ }^{3}$, Samuel Katsuyuki Shinjo ${ }^{1}$
}

1. Universidade de São Paulo, São Paulo (SP), Brazil. 2. Instituto Nacional de Ciencias Médicas y Nutrición Salvador Zubirán, Ciudad de México, Mexico. 3. Hospital San Roque de Gonnet, Buenos Aires, Argentina.

*Corresponding author: pablolivo@yahoo.es

\section{BACKGROUND}

Several studies have shown that the prevalence of interstitial lung disease (ILD) in dermatomyositis (DM) ranges from 17 to $36 \%$. Specifically in Asian patients, this prevalence can be as high as $80 \%$, whereas rapidly progressive ILD (RP-ILD) occurs in $22 \%$ of patients with mortality rate exceeding $50 \%$. Herein, we analyze the frequency of ILD and RP-ILD in a large Latin American sample of patients with DM.

\section{MATERIALS AND METHODS}

This multicenter cross-sectional study, which took place between 2003 and 2020, enrolled adult patients with defined classical DM (EULAR/ACR classification criteria 2017) and clinically amyopathic DM (CADM) (Gerami et al. criteria 2006), following up in tertiary centers from Argentina, Mexico and Brazil. All data were evaluated using electronic medical records with prestandardized and parameterized information. Interstitial lung disease (ILD) was defined as the presence of inflammation and/or healing of the pulmonary interstitial at onset of diagnosis or follow-up of disease), whereas rapidly progressive ILD (RP-ILD) was defined as acute and progressive worsening of dyspnea requiring hospitalization, supplemental oxygen or respiratory failure and subsequent intubation, within three months of ILD diagnosis). Patients with positive anti-Jo-1 antibodies were excluded. All patients requested high-resolution chest tomography during the first 3 months after the suspected diagnosis.

\section{RESULTS}

In total, 270 patients were included: 24, 91 and 155 from Argentina, Brazil and Mexico, respectively. There were 200 (74.1\%) DM and 70 (25.9\%) CADM patients. The mean age at disease onset was $42.8 \pm 14.8$ years, with predominance of female $(70.4 \%)$ and Hispanic ethnicity (49.6\%). The mean time duration between diagnosis and the onset of symptoms was $7.9 \pm 11.2$ months. The general prevalence (at onset of diagnosis and follow-up) of ILD was $25.9 \%$, and the frequency of RP-ILD was $1.5 \%$. There was no statistical difference in the prevalence of ILD and RP-ILD between the DM and CADM patients.

\section{CONCLUSIONS}

Although the frequency of ILD in Latin American patients with DM was comparable that of patients from other regions, there was a low prevalence of RP-ILD in the present study, mainly compared with studies of Asian patients. Our data show that the frequency and severity of lung involvement in DM can vary according to geographic region. Additional studies are needed to evaluate the role and reaction of myositis-specific antibodies (e.g., anti-MDA-5) in Latin American patients with DM. 